ERRATUM

\title{
Molecular detection to improve surgery
}

\section{Gemma K. Alderton}

Nature Reviews Cancer 14, 515 (2014)

The article incorrectly stated the DOI number for the original research paper; it should have been 10.1073/ pnas.1404724111. The error has been corrected in the online version of the article. 